\title{
Urban birth and risk of schizophrenia: a worrying example of epidemiology where the data are stronger than the hypotheses
}

\author{
JOHN MCGRATH and JAMES SCOTT
}

\begin{abstract}
There is robust and consistent evidence from epidemiological studies showing that urban birth is associated with an increased risk of developing schizophrenia. Evidence suggests that this exposure may be associated with a sizeable proportion of cases. To date the candidate exposures underlying the urban birth risk factor have included infectious agents, low prenatal vitamin D, toxins associated with pollution, and stress. However, in general, the hypotheses proposed to explain the urban birth risk factor have been unsatisfying. In light of the general trend towards increasing urbanization, it is feasible that the attributable fraction of schizophrenia associated with urban birth may increase. The psychiatric research community should have a sense of urgency in exploring the mechanisms linking urban birth and risk of schizophrenia.
\end{abstract}

\section{INTRODUCTION}

Links between the urban environment and schizophrenia have long been a topic of interest (Faris \& Dunham, 1939; Freeman, 1994). The fact that the prevalence of schizophrenia appeared greater in the city, especially poorer urban regions, could be easily dismissed as merely a reflection of "social drift" - people with disabling conditions tend to be poor and thus tend to live in areas with cheaper accommodation (Goldberg \& Morrison, 1963). Perhaps the maldistribution of mental health services also could contribute to an apparent urban-rural gradient in the prevalence of serious mental disorders. However, the situation changed abruptly when a series of well-designed studies found an association between urban birth and an increased risk of developing schizophrenia (Lewis et al., 1992; Marcelis et al., 1998; Mortensen et al., 1999). Urban birth and urban residence prior to onset act as a proxy marker for an exposure (or set of exposures) that apparently influences the incidence of the condition.

Over the last eight years the association between urban birth and schizophrenia has been relatively consistent (Pedersen \& Mortensen, 2006; Spauwen \& van Os, 2006). In contrast to many other risk factors for schizophrenia (e.g. influenza, obstetric complications), urban

Address for correspondence: Professor J. McGrath, Queensland Centre for Mental Health Research, The Park Centre for Mental Health, Wacol, QLD 4076 (Australia).

Fax: +61-7-3271.8698

E-mail: john_mcgrath@qcsr.uq.edu.au

Declaration of Interest: The Stanley Medical Research Institute supported this research. birth is a "stubborn" risk factor. The data are relatively consistent in effect size (and direction). Overall, the data are high quality and convincing. In contrast, the hypotheses proposed to explain the finding have not matched the quality of the data.

\section{Mixed results from systematic reviews}

We had the opportunity to examine urbanicity and schizophrenia in two recent systematic reviews. For example, a systematic review reported that those living in cities had significantly higher incidence rates of schizophrenia compared to those living in mixed urban-rural sites (McGrath et al., 2004). These studies, which were drawn from 33 countries, generated a total of 1,456 incidence rates. While there were many studies from mixed urban-rural catchment areas $(n=41)$, there were only 19 studies from "pure" urban sites, and 5 from "pure" rural sites. Nevertheless, when the rates were divided into urban versus mixed urban/rural, urban sites had significantly higher rates for persons. However, these studies did not examine place of birth. The review highlighted a relative paucity of incidence data from rural regions or from the developing world. However, the results from the systematic review are broadly consistent from the more precise within-nation studies conducted in Scandinavia and Europe.

Unexpectedly, when we undertook a systematic review of the prevalence of schizophrenia, we did not find a significant urban-rural gradient (Saha et al., 2005). We identified 73 prevalence estimates from urban sites, 48 estimates from rural sites and 137 from mixed urbanrural sites. While the mixed urban/rural estimates were higher than urban and rural rates, this difference was not statistically significant $\left(F_{2.235}=1.63, p=.20\right)$, nor were 
urban estimates significantly different compared to rural $\left(F_{t, 120}=0.95, p=.33\right)$. In light of (a) the urban-rural gradient in incidence studies, (b) "social drift" theory, and (c) the evidence suggesting less industrialized settings may facilitate recovery (Warner, 1999), the lack of an urban-rural gradient in the prevalence of schizophrenia is hard to explain.

\section{Urban birth is associated with a large population attributable fraction}

While the relative risk associated with urban birth is modest (about two fold risk compared to rural birth), the population attributable fraction is about $30 \%$ (Marcelis et al., 1998; Mortensen et al., 1999). This is a reflection of the high proportion of the population in the developed world that is "exposed" to urban birth. While the interpretation of population attributable fractions requires caution, the notion that nearly a third of all individuals may have schizophrenia as a result of the yet-to-be-identified urban risk factor(s) is startling.

No one needs reminding that schizophrenia contributes substantially to the global burden of disease (Murray \& Lopez, 1996). Also, clinicians and health planners are painfully aware that even with optimal funding and evidence-based treatment, a large portion of the disability associated with schizophrenia is unavoidable (Andrews et al., 2003; 2004). The bottom line is that these two statements synergize into a potent argument for greater investment in research to help prevent schizophrenia. While prevention may seem quixotic, long journeys need to start somewhere. We argue that when setting research priorities, risk factors associated with higher attributable fractions should be given priority. Thus, candidate exposures related to the urban birth risk factor warrant high priority. Even more chilling, population demographics indicate increasing urbanization in both the developed and developing world, (McMichael, 2001). While speculative, it is feasible that the population attributable fraction of schizophrenia associated with urban birth will rise in the decades to come.

\section{Candidate exposures underpinning urban birth}

Epidemiology is a blunt instrument - it is much better at generating candidates than proving them. In the absence of understanding the neurobiological correlates of schizophrenia, it becomes difficult to discriminate between risk-modifying factors (i.e effectors) and riskindicating factors (proxy markers) (McGrath \& Murray, 2003). With respect to urban birth, schizophrenia research has an example where the epidemiological data are much strong than the candidate exposures.

A number of biological candidates have been investigated as effectors for the increased incidence of schizophrenia in those of urban birth. Infection during the prenatal period or during the early years of life could contribute to the urban-rural gradient. Those living in the city could be at increased risk of infection because of overcrowding and the more rapid spread of contagious agents (Torrey \& Yolken, 1998). However, the epidemiological evidence for this is mixed (Agerbo et al., 2001; Wahlbeck et al., 2001). Equally, those living in rural regions may more likely be exposed to farm animals, which could contribute to a different range of infective exposures.

Population levels of vitamin D have clear urban-rural gradients (lower in urban regions) (Nesby-O'Dell et al., 2002). While the evidence from animal models has demonstrated that low prenatal vitamin $\mathrm{D}$ alters brain development (Eyles et al., 2003), there is no direct evidence demonstrating a link between this candidate exposures and the urban-rural gradient in the incidence of schizophrenia. Malnutrition in general has been associated with higher rates of schizophrenia, however it is hard to map this candidate along urban-rural gradients (Hoek et al., 1998).

Toxins related to air pollution have urban-rural gradients, and are thus worthy candidates. It is known that many of these candidates (e.g. lead) can impact on brain development, thus they have good biological plausibility. Pederson has recently examined proximity to major roads as a proxy marker of exposure to fuel-related toxins, however the study found no association between the variables of interest (Pedersen \& Mortensen, 2006a).

Could differential exposure to substance abuse contribute to the urban-rural gradient? Increased exposure to cannabis (Hall et al., 2004; Semple et al., 2005) and other substances seem like reasonable candidates, but once again, there is a relative paucity of the data to suggest that this exposure underlies the increased urban incidence of schizophrenia.

In recent decades there has been a long-overdue renaissance in research examining the role of social stress and risk of schizophrenia (van Os et al., 2005). Childhood stress (Corcoran et al., 2003), early maternal stress (Spauwen et al., 2004), and child abuse (Read et al., 2005), have all been postulated as potential component causes for schizophrenia. Social capital (i.e. the "connectedness" and positive support provided by a close-knit community) (McKenzie et al., 2002) is one broad mechanism that may differentially buffer or protect individuals according to urban-rural gradients. Similarly, 
social fragmentation (as measured by households with non-married adults, adults living alone, population turnover or private renting) is associated with higher first admission rates for psychosis. This association occurred independent of urbanicity (Allardyce et al., 2005 ). These candidates are thought provoking and worthy of investigation (Drukker et al., 2005). Unfortunately, these exposures are also notoriously difficult to partition into units suitable for measurement at the analytic level. Just as well schizophrenia researchers like a challenge!

In summary, the quality of the empirical data linking urban birth and/or urban residence with risk of schizophrenia is relatively strong (and certainly stronger than many candidate genes currently attracting biomedical research funds). In contrast to the strength of the data, the candidate exposures and hypotheses proposed to underpin this gradient are generally unconvincing.

\section{What should be done now?}

First and foremost, the schizophrenia research community needs to generate a sense of urgency about the importance of understanding the urban birth risk factors. We need to wake from our slumber. If a third of all cardiovascular disease had been linked to an environmentally-mediated risk indicator, one would predict that government funding agencies would invest heavily in projects aimed at understanding the mechanisms of action linking the variables of interest (e.g. think of the "epidemic" of cardiovascular disease in the mid-twentieth century and the Framingham Heart study). The fact that this has not yet happened in schizophrenia reflects poorly on our discipline.

As with any field of epidemiology, we need to strike a balance between further replications versus more focused research related to candidate exposures. It would be a great shame if the urban birth risk factor, like season of birth, degenerated into "circular epidemiology" (Kuller, 1999), where an oft-replicated finding can fail to move from descriptive to analytical case-control and longitudinal studies. This has not happened yet, as the replication studies have been able to refine the research strategy and "add value" to the results. For example, some studies have tried to refine the critical window of exposure (Marcelis et al., 1999; Pedersen \& Mortensen, 2001), or examine secular trends in the effect size of the risk factor (Pedersen, 2006). Not all studies in developed societies have detected an association (Haukka et al., 2001). Well designed studies that do not find an association will be important for refining the field of candidate exposures.
Another strategy that warrants closer inspection is to try to "amplify the signal". If urbanicity is a risk factor for schizophrenia, then this should be readily detected in incidence studies in very large cities. Basing studies in Denmark or the Netherlands may not provide a sufficient variation in exposures to allow candidate refinement. Cities in these two nations are relatively small (no cities in the top 300 cities by population currently). Recently, an incidence study from Sao Paulo (the second largest city, 10.3 million), reported a relatively low incidence of schizophrenia (Menezes et al., 2006). Studies such as the Aetiology and Ethnicity in Schizophrenia and Other Psychoses (AESOP) (Morgan et al., 2005) have the capacity to examine how incidence varies between cities, which could also help guide candidate selection.

As candidate exposures are generated, analytical epidemiology can test more precise hypotheses. For example, if prenatal exposures such as infection, lead or vitamin D deficiency are implicated, then the assessment of biological specimens (banked maternal sera, neonatal dried whole blood spots) could examine the putative association directly. Animal studies can also help by assessing the biological plausibility of different candidates (e.g. fever due to infection, social defeat, stressors). Candidate exposures can then be rank ordered on a league table to help set research priorities. Furthermore, within the field of candidate risk factors for schizophrenia, we argue that candidates that offer the potential for public health interventions should also be accorded priority (McGrath, 2003).

\section{CONCLUSION}

We have a clear and strong signal telling us urban birth and urban residence increases the risk of developing schizophrenia. However, the quality of the data has not been matched by the quality of the hypotheses. Currently we have a few candidate exposures, but we need more. The response to testing of these candidate exposures has generally been underwhelming. Let's hope that the next decade of research on urbanicity and schizophrenia will be less ataxic and more focused. Schizophrenia researchers can not afford to squander good data.

\section{REFERENCES}

Agerbo E., Torrey E.F. \& Mortensen P.B. (2001). Household crowding in early adulthood and schizophrenia are unrelated in Denmark: a nested case-control study. Schizophrenia Research 47, 243-246. 
Allardyce J., Gilmour H., Atkinson J., Rapson T., Bishop J. \& McCreadie R.G. (2005). Social fragmentation, deprivation and urbanicity: relation to first-admission rates for psychoses. British Journal of Psychiatry 187, 401-406.

Andrews G., Sanderson K., Corry J., Issakidis C. \& Lapsley H. (2003). Cost-effectiveness of current and optimal treatment for schizophrenia. British Journal of Psychiatry 183, 427-435.

Andrews G., Issakidis C., Sanderson K., Corry J. \& Lapsley H. (2004). Utilising survey data to inform public policy: comparison of the cost-effectiveness of treatment of ten mental disorders. British Journal of Psychiatry 184, 526-533.

Corcoran C., Walker E., Huot R., Mittal V., Tessner K., Kestler L. \& Malaspina D. (2003). The stress cascade and schizophrenia: etiology and onset. Schizophrenia Bulletin 29, 671-692.

Drukker M., Buka S.L., Kaplan C., McKenzie K. \& van Os J. (2005). Social capital and young adolescents perceived health in different sociocultural settings. Social Science and Medicine 61, 185-198.

Eyles D., Brown J., Mackay-Sim A., McGrath J. \& Feron F. (2003). Vitamin D3 and brain development. Neuroscience 118, 641-653.

Faris R.E.L. \& Dunham H.W. (1939). Mental Disorders in Urban Areas. Chicago University Press: Chicago.

Freeman H. (1994). Schizophrenia and city residence. British Journal of Psychiatry, Suppl. 23, 39-50.

Goldberg E.M. \& Morrison S.L. (1963). Schizophrenia and social class. British Journal of Psychiatry 109, 785-802.

Hall W., Degenhardt L. \& Teesson M. (2004). Cannabis use and psychotic disorders: an update. Drug and Alcohol Review, 433-443.

Haukka J., Suvisaari J., Varilo T. \& Lonnqvist J. (2001). Regional variation in the incidence of schizophrenia in Finland: a study of birth cohorts born from 1950 to 1969. Psychological Medicine 31, 10451053.

Hoek H.W., Brown A.S. \& Susser E. (1998). The Dutch famine and schizophrenia spectrum disorders. Social Psychiatry and Psychiatric Epidemiology 33, 373-379.

Jablensky A.V. \& Kalaydjieva L.V. (2003). Genetic epidemiology of schizophrenia: phenotypes, risk factors, and reproductive behavior. American Journal of Psychiatry 160, 425-429.

Kuller L.H. (1999). Circular epidemiology. American Journal of Epidemiology 150, 897-903.

Lewis G., David A., Andreasson S. \& Allebeck P. (1992). Schizophrenia and city life. Lancet 340, 137-140.

Marcelis M., Navarro-Mateu F., Murray R., Selten J.-P. \& van Os J. (1998). Urbanization and psychosis: a study of 1942-1978 birth cohorts in The Netherlands. Psychological Medicine 28, 871-879.

Marcelis M., Takei N. \& van Os J. (1999). Urbanization and risk for schizophrenia: does the effect operate before or around the time of illness onset? Psychological Medicine 29, 1197-1203.

McGrath J.J. (2003). Invited commentary: Gaining traction on the epidemiologic landscape of schizophrenia. American Journal of Epidemiology 158, 301-304.

McGrath J.J. \& Murray R.M. (2003). Risk factors for schizophrenia: from conception to birth. In Schizophrenia (ed. D.R. Weinberger and S.R. Hirsch), pp. 232-250. Blackwells: Oxford.

McGrath J., Saha S., Welham J., El Saadi O., MacCauley C. \& Chant D. (2004). A systematic review of the incidence of schizophrenia: the distribution of rates and the influence of sex, urbanicity, migrant status and methodology. BMC Medicine 2, 13.

McKenzie K., Whitley R. \& Weich (2002). Social capital and mental health. British Journal of Psychiatry 181, 280-283.

McMichael A.J. (2001). Human Frontiers, Environments and Disease. Cambridge University Press: Cambridge.
Menezes P., Scazufca M., Casgrande K., Schlithler A.C., Cordeiro D., Coutinho L.M., Busatto G., McGuire P., Fearon P. \& Murray R.M. (2006). Incidence of first contact psychosis in Sao Paulo, Brazil. Schizophrenia Research 81, 173.

Morgan C., Mallett R., Hutchinson G., Bagalkote H., Morgan K., Fearon P., Dazzan P., Boydell J., Harrison G., Murray R., Jones P., Craig T. \& Leff J. (2005). Pathways to care and ethnicity. I. Sample characteristics and compulsory admission: a report from the AESOP study. British Journal of Psychiatry 186, 281-289.

Mortensen P.B., Pedersen C.B., Westergaard T., Wohlfahrt J., Ewald H., Mors O., Andersen P.K. \& Melbye M. (1999). Effects of family history and place and season of birth on the risk of schizophrenia. New England Journal of Medicine 340, 603-608.

Murray C.J. \& Lopez A.D. (1996). The Global Burden of Disease. Harvard School of Public Health: Boston.

Nesby-O'Dell S., Scanlon K.S., Cogswell M.E., Gillespie C., Hollis B.W., Looker A.C., Allen C., Doughertly C., Gunter E.W. \& Bowman B.A. (2002). Hypovitaminosis D prevalence and determinants among African American and white women of reproductive age: third National Health and Nutrition Examination Survey, 19881994. American Journal of Clinical Nutrition 76, 187-192.

Pedersen C.B. (2006). No evidence of time trends in the urban-rural differences in schizophrenia risk among five million people born in Denmark from 1910 to 1986. Psychological Medicine 36, 211-219.

Pedersen C.B. \& Mortensen P.B. (2001). Evidence of a dose-response relationship between urbanicity during upbringing and schizophrenia risk. Archives of General Psychiatry 58, 1039-1046.

Pedersen C.B. \& Mortensen P.B. (2006a). Urbanization and traffic related exposures as risk factors for schizophrenia. BMC Psychiatry 6, 2 .

Pedersen C.E \& Mortensen P.B. (2006b). Why factors rooted in the family may solely explain the urban-rural differences in schizophrenia risk. Epidemiologia e Psichiatria Sociale 15, 247-251.

Read J., van Os J., Morrison A.P. \& Ross C. A. (2005). Childhood trauma, psychosis and schizophrenia: a literature review with theoretical and clinical implications. Acta Psychiatrica Scandinavica 112, 330350.

Saha S., Chant D., Welham J. \& McGrath J. (2005). A systematic review of the prevalence of schizophrenia. PLOS Medicine 2, e141.

Semple D.M., McIntosh A.M. \& Lawrie S.M. (2005). Cannabis as a risk factor for psychosis: systematic review. Journal of Psychopharmacology 19, 187-194.

Spauwen J. \& van Os J. (2006). The psychosis proneness. Psychosis persistence model as an explanation for the association between urbanicity and psychosis. Epidemiologia e Psichiatria Sociale 15 , 252-257.

Spauwen J., Krabbendam L., Lieb R., Wittchen H.-U. \& van Os J. (2004). Early maternal stress and health behaviours and offspring expression of psychosis in adolescence. Acta Psychiatrica Scandinavica 110, 356-364.

Torrey E.F. \& Yolken R.H. (1998). At issue: is household crowding a risk factor for schizophrenia and bipolar disorder? Schizophrenia Bulletin 24, 321-324.

van Os J., Krabbendam L., Myin-Germeys I. \& Delespaul P. (2005). The schizophrenia envirome. Current Opinion in Psychiatry 18, 141-145.

Wahlbeck K., Osmond C., Forsen T., Barker D.J. \& Eriksson J.G. (2001). Associations between childhood living circumstances and schizophrenia: a population-based cohort study. Acta Psychiatrica Scandinavica 104, 356-360.

Warner R. (1999). Schizophrenia and the environment: speculative interventions. Epidemiologia e Psichiatria Sociale 8, 19-34. 